\title{
Temperature and Humidity Memory Instrument And Its Data Processing System
}

\author{
D.D. Chi \\ Department of Mechanical and Electrical Engineering \\ Tangshan Vocational College of Science and Technology \\ Tangshan, P.R. China
}

\begin{abstract}
The instrument uses a single chip relative humidity and temperature multi sensor SHT11 module comprising a calibrated digital output, which can measure temperature and relative humidity of environment at any moment, timely and real time. It displays temperature-humidity value, dew point value, date and time at the same time by lattice graphics Liquid Crystal Display Module (LCM) HGO12864. Temperature-humidity value and relevant time are saved in nonvolatile Ferroelectric Random Access Memory (FRAM) FM31256, which can assure data do not lose after power of system fail. Real-time data or data in FM31256 can be upload PC by USB interface, and then is protracted temperature-humidity history curve or real-time curve by internet applications. It can not only calculate maximum, minimum, average and variance among random measuring period of time but also make further statistics and analysis, report forms printing, and setup basic measure parameters of instrument. Its power supply is $3.6 \mathrm{~V}$ Li-battery or $220 \mathrm{~V}$ Alternating Current(AC). It is low power-consuming, high precision, plug and play and better universal performance.
\end{abstract}

Keywords: temperature-humidity; USB interface; FRAM

\section{INTRODUCTION}

In many fields, such as industry, agriculture, public place, laboratory, research room, greenhouse, library, factory building, the temperature-humidity needs measuring and controlling continuously. For example, heavy humidity causes to go mouldy and go bad in grain, medicines, cigarette and tea warehouse. If temperature and humidity in cotton warehouse are unsuitable, spontaneous combustion will take place in it. Performance of precision instrument and semiconductor device is reduced because of superheating. So the temperature and humidity are two very important physics parameters. But traditional temperature-humidity measuring instrument adopts the method of measuring the temperature and humidity separately, which can't well get rid of the interference of temperature when measuring humidity. In addition, the interchange ability of sensor is bad, the circuit is complicated, calibration time is long, and complicated [1].

The instrument adopts late-model temperature-humidity integration digital output sensor---SHT11, which has high measurement accuracy, good Long-term stability and fast response time. Data acquired by SHT11 not only can be uploaded directly to PC through USB interface but also can be keep in nonvolatile FRAM---FM31256, which can not lose even if the system is power-fail. At the same time, temperature, humidity and dew point value, now time and date are displayed real time in lattice graphics Liquid crystal display(LCD), which makes user can get temperature and humidity information more expediently. The power of instrument is rechargeable lithium battery or $220 \mathrm{~V}$ AC, which has a lot of advantages such as low consumed power, small volume, light weight and good portability. More important, communication between the instrument and PC has replaced traditional RS232 serial interface by double-mode USB interface (USB-HOST and USB-DEVICE), which can be implemented not only directly but also indirectly through flash disk. So the instrument has small interface, plug and play and better universal performance.

\section{THE STRUCTURE OF THE INSTRUMENT}

The instrument regards AT89LV52 as the core, which is made up of data acquisition module, data memory module, USB communication module, data processing and analysis module in PC. It not only can acquire temperature and humidity value or stop at any time, but also can acquire them and stop through timing routine that the corresponding application software of the instrument set acquisition Initial time, stop time and time interval. In addition, it can also set up memory mode, upper and lower limit value of temperature and humidity to alarm and now time and date. The gross structure of the instrument is as shown in Fig.1.

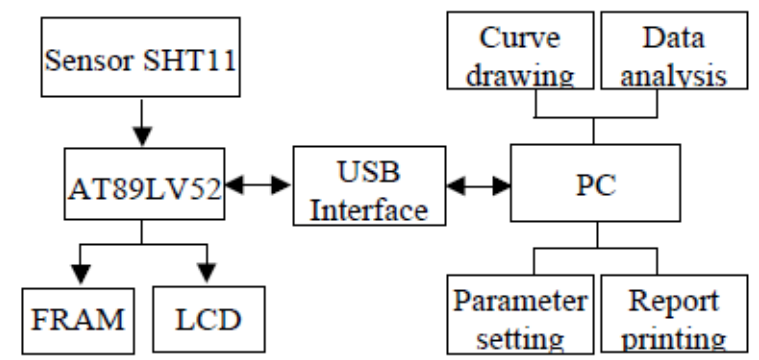

FIGURE I. THE BLOCK DIAGRAM OF THE INSTRUMENT GROSS STRUCTURE.

\section{A. Data Acquisition Module}

The instrument uses a temperature-humidity integration digital output sensor---SHT11, which integrate temperature and humidity response element, signal amplifier, A/D converter, One Time Programmable Read Only Memory (OTP ROM), I2C bus etc peripheral circuit. It outputs directly digital signal of the relative humidity and temperature after calibration. It strengthens signal intensity and anti-interference and long-time stability, which resolves availably deficiency of traditional temperature and humidity sensor. In addition, it can also measure dew-point accurately, which will not introduce error 
because of the temperature difference of temperature and humidity [2].

The instrument can acquire accurate data by adopting method of software to compensate non-linearity of humidity sensor. The formula to amend humidity value is as follows:

$$
\mathrm{RH}_{\text {linear }}=\mathrm{C}_{1}+\mathrm{C}_{2} \times \mathrm{SO}_{\mathrm{RH}}+\mathrm{C}_{3} \times \mathrm{SO}_{\mathrm{RH}} \times \mathrm{SO}_{\mathrm{RH}}
$$

In the above formula, SORH is relative humidity measuring value of the sensor, which is as follows (the humidity can select from 14 or 12 bit, the temperature can select from 12 or 8 bit):

$$
\begin{aligned}
& 12 \text { bit } \mathrm{SO}_{\mathrm{RH}}: \mathrm{C}_{1}=-4, \mathrm{C}_{2}=0.0405, \mathrm{C}_{3}=-2.8 \times 10^{-6} \\
& 8 \text { bit } \mathrm{SO}_{\mathrm{RH}}: \mathrm{C}_{1}=-4, \mathrm{C}_{2}=0.648, \mathrm{C}_{3}=-7.2 \times 10^{-4}
\end{aligned}
$$

The above formula is used to calculate humidity value in the case of 25 degree, but the real measuring temperature is changed within the specific range, so temperature coefficient of humidity sensor should be considered, which can be compensated according to the following formula:

$$
R H_{\text {true }}=(T-25) \times\left(t_{1}+t_{2} \times S O_{R H}\right)+R H_{\text {linear }}
$$

In the above formula, $\mathrm{T}$ is actual temperature value. Its coefficient is as follows:

$$
12 \text { bit } \mathrm{SO}_{\mathrm{RH}}: \mathrm{t}_{1}=0.01, \mathrm{t}_{2}=0.00008
$$

8 bit SORH: $\mathrm{t} 1=0.01, \mathrm{t} 2=0.00128$

\section{B. Data Memory and Display Module}

The instrument adopts advanced nonvolatile FRAM--FM31256, which has 10 years retention time after power-fail, unlimited read/write endurance. So it assures historical data memory reliability, and then they can be done cure-drawing, analysis, processing, report-printing, etc. In addition, the memory integrates real-time clock (RTC), low-VDD reset circuit (instead of separate clock chip---DS1302) and watchdog timer circuit (instead of X5045 chip), which makes the volume of the instrument more smaller, higher integration and better portability. Interface circuit between it and SCM AT89LV52 is as shown in Fig.2. Its pin12 and pin13 joint with P2.1 and P2.2 of SCM separately. Its pin5 joints with INT1 of SCM to input interrupt signal, which can make the instrument realize early power-fail warning function. Its pin6 to activelow reset output for VDD and watchdog outputs reset signal to the whole system, which can assure the integrality that inter programme runs [3] Display module adopts low consumed power and low voltage HGO12864 module, which can display temperature, humidity, dew point, now time and data together, so that it can make users watch more conveniently. The LCM adopts LED blue back lighting what is controlled by key, which can make users read temperature and humidity value under dim-beam occasion.

\section{USB Communication Module}

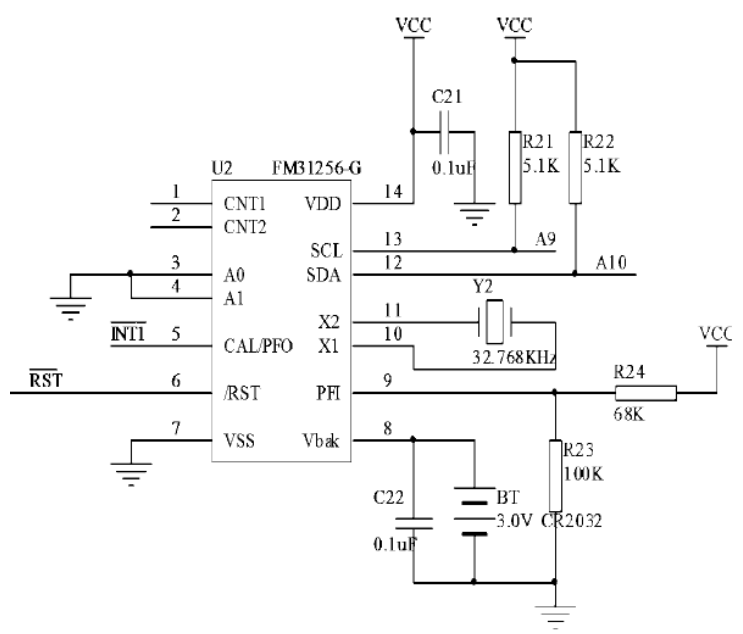

FIGURE II. THE INTERFACE CIRCUIT DIAGRAM BETWEEN FM31256 AND AT89LV52.

USB interface has got extensive popularization and application with a great deal of characteristics such as its plug and play, convenience, apt expanding and high speed. But, to developers, researching USB interface is not a easy thing, because it requires developers to comprehend deeply USB standard, firmware programme and driver compiling, which restrains general hardware engineer's development to USB interface products[4]. But, in the system, universal USB interface chip CH375 is used. It only needs developers to program sigle-chip and PC application software, so it has many characteristics such as apt using, short development periods. More important, it supports USB-HOST mode and USB-DEVICE mode.

The instrument has broken through traditional single USB external device and adopts double USB plug seat mode, which enable it not only can work in USB-DEVICE mode but also be used in USB-HOST mode, so it provides more convenience to different users and occasions[5]. The interface circuit of CH375 and SCM is as shown in Fig.3.

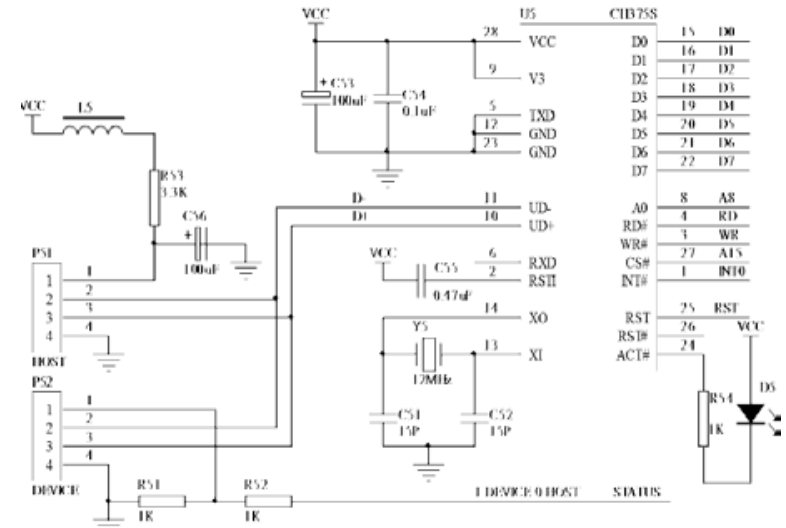

FIGURE III. THE INTERFACE CIRCUIT DIAGRAM BETWEEN CH375 AND SCM.

SCM judges which USB plug seat is working by high or low voltage of STATUS signal, and then makes sure which communication mode the instrument is working in. If the port 
P52 joints with PC, CH375 works in device mode. If the port P51 joints with USB device (for example flash disk), CH375 works in host mode. The both can't work at the same time. Under the idle situation, STATUS signal is low level, SCM makes CH375 works in the host mode. When USB apparatus inserts P51, CH375 will inform automatically SCM to deal with corresponding work. When the P52 joints with USB port of PC, $5 \mathrm{~V}$ voltage of PC makes the STATUS become low level, and then SCM monitors the STATUS signal to switch the CH375 to device mode [6, 7].

The double mode USB interface makes the instrument more universal, more convenient and more portable.

\section{Data processing and Analysis Module in PC}

The PC application software adopts visual programming language---Visual Basic to program. It can realize the following functions: temperature and humidity data curve drawing, showing maximum, minimum and mean in any time interval, to transfer Microsoft Office Excel to display data in list, data statistic analysis and report printing. In addition, the software can set many parameters of the instrument (such as start and stop time, time interval, startup mode, memory mode, warning threshold of temperature and humidity value etc.). The gross structure of the software interface is as shown Fig.4.

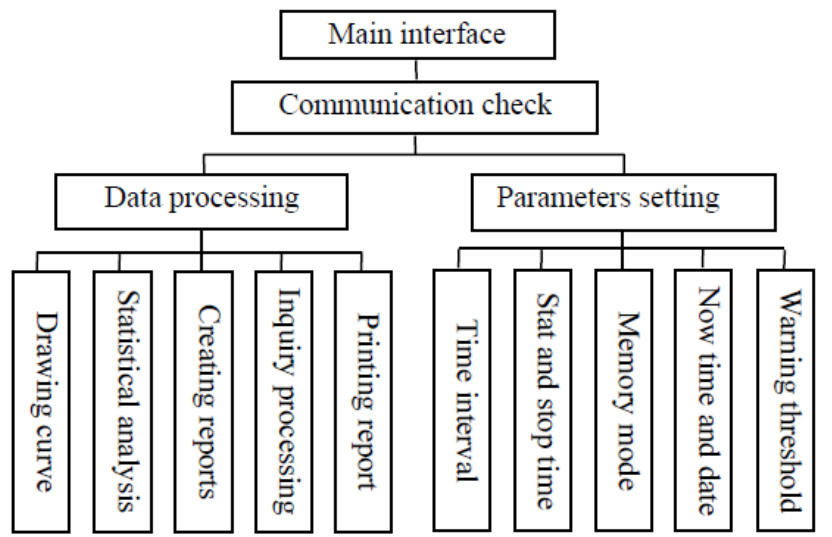

FIGURE IV. THE INTERFACE STRUCTURE AND FUNCTION BLOCK DIAGRAM OF PC APPLICATION SOFTWARE.

III. THE EXPERIMENTAL RESULT AND ITS ANALYSIS

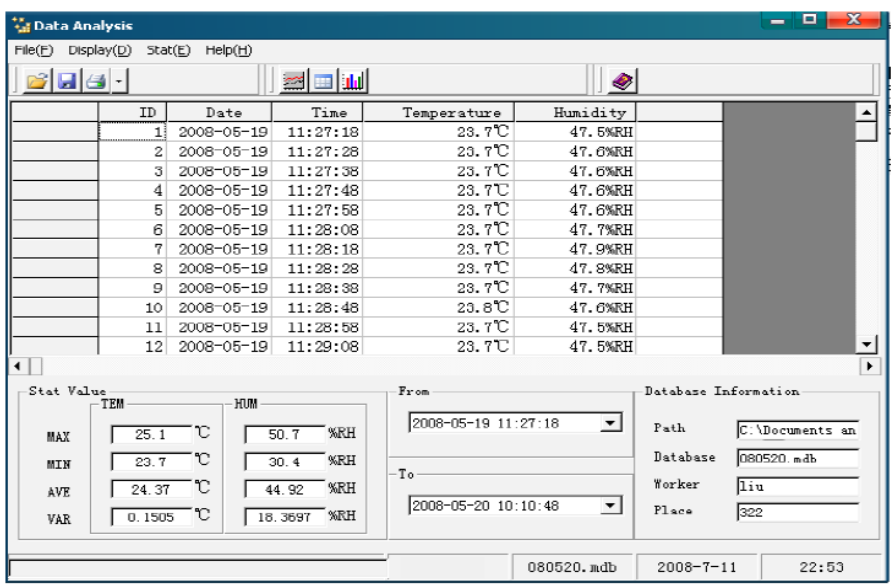

FIGURE V. THE DATA LIST AND ANALYSIS INTERFACE.
The instrument has very good measuring effect in experiment test. In the Fig.5, partial data measured in the greenhouse is shown. Measuring time interval of these data is 3 second. In these data, temperature error does not exceed \pm 0.4 and humidity error does not exceed $\pm 3.0 \%$ through comparing and analyzing with the standard temperature instrument and hygrometer. Nonvolatile memory can store more than 16000 group data. When the instrument is used under the environment which temperature and humidity change slowly, it can measure for about 220 days continuously by gathering once at intervals of every 20 minutes.

\section{CONCLUSION}

The instrument uses advanced single bus sensor, which is reliable, stable, fully calibrated and fully interchangeable. It not only can acquire and memory temperature and humidity data automatically long-time but also can do write-protect to these data that is acquired. In addition, these data can not lose after power-fail because of nonvolatile FRAM. More important, the instrument not only can acquire data automatically according to start time, stop time and time interval set by users, which need not monitoring artificially and is suitable for many dangerous occasion, but also realizes instant start and stop for temperature and humidity acquisition by a key, which can make users more convenient and reduce dependence on the PC. The double mode USB interface makes data transmission more convenient and fast. It can copy data to lab PC by flash disk under the locale without PC. The instrument has many characteristics such as high precision, good stability, high dependability etc.

\section{REFERENCES}

[1] Xu Ping, Tian Yu, et al. "Research of universal temperature and humidity measurement”, China Instrumentation, 2004, (1), pp. 35-37.

[2] Meng Chen, Li Min, et al. "Digital Temperature and Humidity Sensor SHT11 Based on $\mathrm{I}^{2} \mathrm{C}$ Bus and Its Application in the Single-chip Microcomputer System”, International Electronic Elements, 2004,(2), pp. 50-52.

[3] Du Zhongliang, Zhang Xuhui. "Application of FM31256 in Multi-user Multi-rate Watt-hour Meter”, Power System Engineering, 2005, 21 (4), pp. 64.

[4] Zhao Xiaoshun, Yu Huali,et al. "Design of double-mode USB interface of temperature humidity measuring apparatus", Journal of Agricultural Mechanization Research, 2007, (10), pp. 171-173.

[5] Zhao Xiaoshun, Liu Shuxia. "Intelligent network temperature and humidity measuring system based on USB interface”, Proceedings of 2007 International Conference on Agriculture Engineering, 2007, (10), pp. $475-479$.

[6] H. Li, "Design of a Virtual Instrument with USB Interface Based on Chip CH375", Journal of China Three Gorges University (Natural Sciences), 2005, 27 (2), pp. 168-170.

[7] Li Xinlong, Zhai Hongfan, et al. “USB chip CH375's application in a power measurement system”, Control \& Automation, 2006, 22 (9), pp. 310-312. 